\title{
Determining the Effect of Molecular Oxygen Tension on Islet Metabolism and Endothelial Cell Morphology Using a Microfluidic Device
}

\author{
Krishana Sankar*, J.V. Rocheleau* \\ * Department of Physiology, University of Toronto, Ontario, CANADA
}

Donor islets are injected into the portal vein of a recipient to treat type 1 diabetes. The donor tissue eventually becomes lodged in the presinusoidal capsule of the liver. Hypoxia and limited nutrients at this site result in endothelial cell (EC) death and beta-cell dysfunction until the islets can become revascularized with the recipient. Studies in other tissues have shown that hypoxia preconditioning and flow in vitro enhances cell survival and viability post transplantation.

To test the combined effect of preconditioning and flow on pancreatic islets, we exposed 10-12 week old C57BL/6 mouse islets to a hypoxia mimetic $\left(100 \mu \mathrm{M} \mathrm{CoCl}_{2}\right)$ and flow in a microfluidic device. This microfluidic device also allows us to culture pancreatic islets with media flow followed subsequently by measurement of the tissue using immunofluorescence labeling (PECAM-1), live cell fluorescence imaging $\left(\mathrm{NAD}(\mathrm{P}) \mathrm{H}, \mathrm{Ca}^{2+}\right)$, and effluent analysis (VEGF, insulin).

EC connectivity measured using PECAM-1 immunofluorescence increased significantly (1.79- and 2.87-fold) in flow and $\mathrm{CoCl}_{2}+$ flow, respectively. VEGF secretion increased in islets treated with $\mathrm{CoCl}_{2}$ alone and $\mathrm{CoCl}_{2}+$ flow, but decreased from islets treated with flow alone. The NAD(P)H response in beta-cell mitochondria and cytoplasm was unaffected by flow or hypoxia; however, we observed a small 1.28-fold ( $\mathrm{p}<0.02$ ) increase in cytoplasmic $\mathrm{NAD}(\mathrm{P}) \mathrm{H}$ of islets in $\mathrm{CoCl}_{2}+$ flow. Overall, our results show that a combination of $\mathrm{CoCl}_{2}$ and flow enhances $\mathrm{EC}$ area and connectivity due to increased endogenous VEGF secretion. Our data suggest that $\mathrm{CoCl}_{2}$ and $\mathrm{CoCl}_{2}+$ flow increases glucose metabolism in the cytoplasm, which agrees with the shift in metabolism reported in the literature. Overall, our data shows that preconditioning improves EC morphology and increases glycolytic metabolism. Further studies will determine and the effect on beta-cell insulin secretion. 